\title{
Vegetation Database of Oak Forests of Romania
}

\author{
Adrian Indreica
}

\begin{abstract}
This report presents the content of a database with phytosociological relevés of oak and mixed oak forests from Romania (Vegetation Database of Oak Forests of Romania, GIVD ID EU-RO-005). This includes vegetation-plots in which oak species (Quercus robur s.1., Q. petraea s.1., Q. cerris, Q. frainetto, Q. pubescens s.1.), hornbeam (Carpinus betulus) or lime (Tilia cordata, T. tomentosa) are dominants or co-dominants. So far, 4,398 relevés are stored in TURBOVEG format, using 160 sources - published works, $\mathrm{PhD}$ thesis and records of research projects. It is estimated that this sources cover $70-75 \%$ of the total relevés available of this kind. Except species data, $65-85 \%$ of relevees have basic environmental information and all have geographical coordinates - mostly derived from localities or toponyms. Its development started from the need of a unified classification of forest vegetation at a national level, but also with the hope that it could serve for a regional revision of syntaxonomy. So far, using the information from this database, two papers were published and other two are planned.
\end{abstract}

Keywords: oak forest; Quercus; vegetation classification.

\begin{tabular}{|c|c|}
\hline GIVD Database ID: EU-RO-005 & Last update: $2012-07-11$ \\
\hline \multicolumn{2}{|c|}{ Vegetation Database of Oak Forests of Romania } \\
\hline \multicolumn{2}{|c|}{ Scope: A database of phytosociological relevés of oak and mixed oak forests in Romania, including hornbeam or lime dominated forests. } \\
\hline Status: ongoing capture & Period: $1928-2011$ \\
\hline \multicolumn{2}{|c|}{ Database manager(s): Adrian Indreica (adrianindreica@unitbv.ro) } \\
\hline \multicolumn{2}{|l|}{ Owner: (private) } \\
\hline \multicolumn{2}{|l|}{ Web address: [NA] } \\
\hline Availability: after blocking period & Online upload: no \\
\hline Database format(s): TURBOVEG, Excel & Export format(s): TURBOVEG \\
\hline \multicolumn{2}{|l|}{ Publication: none } \\
\hline Plot type(s): normal plots & Plot-size range: $100-2,500 \mathrm{~m}^{2}$ \\
\hline Non-overlapping plots: 4,398 & Estimate of existing plots: 6,000 \\
\hline Total plot observations: 4,398 & Number of sources: 160 \\
\hline \multicolumn{2}{|l|}{ Countries: RO: $100.0 \%$} \\
\hline \multicolumn{2}{|c|}{ Forest: $100 \%$ - Non-forest: aquatic: $0 \%$; semi-aquatic: $0 \%$; arctic-alpine: $0 \%$; natural: $0 \%$; semi-natural: $0 \%$; anthropogenic: $0 \%$} \\
\hline \multicolumn{2}{|c|}{ Guilds: all vascular plants: $100 \%$; bryophytes (terricolous or aquatic): $25 \%$} \\
\hline \multicolumn{2}{|c|}{ Environmental data: altitude: $67 \%$; slope aspect: $85 \%$; slope inclination: $85 \%$; microrelief: $5 \%$; soil depth: $3 \%$; soil pH: $1 \%$} \\
\hline \multicolumn{2}{|l|}{ Performance measure(s): cover: $100 \%$} \\
\hline \multicolumn{2}{|c|}{$\begin{array}{l}\text { Geographic localisation: GPS coordinates (precision } 25 \mathrm{~m} \text { or less): } 13 \% \text {; point coordinates less precise than GPS, up to } 1 \mathrm{~km}: 13 \% \text {; small grid } \\
\text { (not coarser than } 10 \mathrm{~km}): 65 \% \text {; political units or only on a coarser scale }(>10 \mathrm{~km}): 9 \%\end{array}$} \\
\hline $\begin{array}{l}\text { Sampling periods: } 1920-1929: 0.1 \% ; 194 \\
\text { 11.0\%; 2000-2009: } 28.0 \% ; 2010-2019: 13 .\end{array}$ & 949: 0.3\%; 1950-1959: 0.6\%; 1960-1969: 14.0\%; 1970-1979: 26.0\%; 1980-1989: 7.0\%; 1990-1999: \\
\hline Information as of 2012-07-1 & urther details and future updates available from http://www.givd.info/ID/EU-RO-005 \\
\hline
\end{tabular}

Adrian Indreica (adrianindreica@unitbv.ro)

Department of Silviculture, Transilvania University of Brasov, 1, Sirul Beethoven, Brasov, ROMANIA 